\title{
船舶分段多工位协同制造系统数学模型研究及应用*
}

\author{
张 倩 ${ }^{1,2}$ 单忠德 ${ }^{1,2}$ 吴 乐 ${ }^{1,2}$ 王绍宗 ${ }^{1,2}$ 田 媛 ${ }^{1,2}$
}

(1. 北京机科国创轻量化科学研究院有限公司 北京 100083 ;

2. 先进成形技术与装备国家重点实验室 北京 100083)

\begin{abstract}
摘要：针对船舶分段制造生产过程中存在的生产计划柔性差、异常信息处理不及时、系统变化响应速度慢等问题，提出针对 生产过程工位级的协同控制系统, 通过开展协同模型分析, 多工位间协同动态调整机制, 构建多工位协同系统制造数学模型, 采用案例对协同结果进行验证。通过应用多工位协同数学模型可有效提高生产计划精度和柔性, 实现船舶制造车间互联互通, 对提高船舶制造生产效率具有重要意义。
\end{abstract}

关键词: 互联互通; 多工位协同制造系统; 数学模型; 动态协同机制

中图分类号: U673

\section{Research and Application of Mathematical Model of Ship Sectional Multi-station Cooperative Manufacturing System}

\author{
ZHANG Qian $^{1,2}$ SHAN Zhongde ${ }^{1,2}$ WU Le $^{1,2} \quad$ WANG Shaozong $^{1,2} \quad$ TIAN Yuan $^{1,2}$ \\ (1. Advanced Manufacture Technology Center, China Academy of Machinery Science \& Technology, \\ Beijing 100083;
}

2. State Key Laboratory of Advanced Forming Technology and Equipment, Beijing 100083)

\begin{abstract}
Aiming at the problems of poor flexibility of production planning, untimely processing of abnormal information and slow response to system changes in the production process of ship sectional manufacturing, a cooperative control system for the production process is proposed. Through the analysis of collaborative model, the dynamic adjustment mechanism of multi-station collaboration, the manufacturing mathematical model of multi-station collaborative system is built, and the collaborative results are verified by case listing. The application of multi-station collaborative mathematical model can effectively improve the accuracy and flexibility of production planning, realize the interconnection of shipbuilding workshops, and is of great significance to improve the production efficiency of shipbuilding.
\end{abstract}

Key words: interconnection; multi-station cooperative manufacturing system; mathematical model; dynamic collaboration mechanism

\section{0 前言}

船舶制造过程是典型的离散制造, 急需开展数 字化设计、综合管控和船舶建造的信息化，从而全 面提高船舶制造的质量和效率, 降低制造成本, 提 升船舶产品的竞争力。“数字化造船” [1] 就是将计算 机技术、先进造船技术、信息技术、网络技术等综 合应用于船舶产品的全流程, 实现生产过程中物料 信息、工艺信息、生产进程、质量控制、生产管控

\footnotetext{
* 高技术船舶科研资助项目(MC-201618-Z01-03)。20190924 收到初稿,
} 20191226 收到修改稿
等数字化表达, 实现生产过程的柔性、均衡、高效 生产的目的, 从而使造船企业获取最大的经济效益。 高技术船舶与海洋工程装备领域的数字化、信 息化发展迅速。在船舶数字化方面开展大量的研究 与应用工作 ${ }^{[2-4]}$ 。在切割、中小组立焊接，分段车间 看板系统、能源集中采集管控, 工艺装备自动化等 方面开展数字化研究; 船舶企业信息化, 互联互通 应用研究, 不同阶段的实施 PDM 系统、ERP 系统 和 MES 系统研究 ${ }^{[5]}$, 提高了船舶制造过程的生产计 划、物流、质量等的数字化、信息化水平。我国 “数 字化造船” 经过十多年的发展, 已具备较好的信息 化基础, 但是实现互联互通等制约船舶智能制造的 
关键技术依然存在。

船舶制造车间互联互通信息化过程中, 目前针 对车间工位级的生产计划管理仍多采用传统的计划 下达后人工管理, 车间工位级生产过程的数字化技术 及系统研究相对较少。为进一步提高船舶制造车间生 产效率, 本文提出针对分段制造过程的多工位协同 制造系统 (Multi-station cooperative manufacturing system, MSCMS), 基于现有工位级生产过程, 分 析影响工位级船舶生产效率相关因素, 建立多工位 协同模型，并采用案例进行了验证分析。

\section{1 多工位协同制造分析}

目前在船舶制造过程车间级信息化方面，车间 计划编制等方面开展了数字化技术研究 ${ }^{[6-10]}$, 对于 车间工位计划执行以及多个并行工位的计划协同、 工位执行反馈研究相对较少, 多工位间的协同作业 机制研究不够; 当生产工位出现异常, 生产过程中 不能柔性的及时协同调整; 同时也会出现工位任务 忙闲不均, 存在工位急工、停工时间长, 造成生产 过程效率低下, 开展多工位协同技术研究工位级别 柔性制造有重要意义。

要实现船舶制造车间级各工位间的协同制造过 程, 首先需要分析车间现有的生产模式以及工位级 生产过程中存在的问题。

(1) 船舶制造生产计划一般包括年度计划、季 度计划和月度计划, 现有的车间生产计划按照月度 计划下达到车间, 针对工位级生产过程而言, 月度 计划颗粒度大, 无法具体的为每个工位提供精确的 生产计划, 导致多个工位间的信息不对称, 加工步 调及节奏难以达到一致，生产效率较低。

以分段制造为例, 船舶制造过程的切割一加工 工位中, 切割后的零件需要进行理料配材、坡口加 工、自由边打磨、曲加工等, 作为中小组立工位的 生产物料。生产制造中按照月度作业下达生产计划, 钢料加工车间计划却没有合适的系统或者手段为每 个工位提供每日作业计划, 切割一加工工位间物料加 工的步调及节奏难以达到一致, 导致生产过程中停工/ 急工频繁，常常造成组立工位生产计划的延迟。

(2) 现有车间工位级的生产管理方式仍采用人 工管理, 针对实际生产过程中出现的紧急插单任务、 计划变更、设备故障、生产资源不足等各种异常情 况, 无法做到及时响应, 导致计划延误, 无法按时 完成生产。

目前我国船厂为提高生产效率, 在资源配置与
负荷计算上, 通常采用增加切割一加工工位的中间 产品缓冲量的方法降低影响。对于计划变更也只能 人工管理计划调整, 响应时间比较长。

针对上述存在的问题, 为解决切割一加工一中 小组立工位之间的工位间信息不对称、工位节奏不 一致等问题, 在实现车间生产过程中工位、设备、 物料、环境、班组等感知一组网一传输基础上, 研 究基于串并联多工序多工位加工系统的离散动态系 统建立产品的传递状态数学模型, 掌握多工序内部 和工位间的生产节奏，确保在生产指令下物资、人 员、工具在不同工位的合理分配与流转, 为优化调 度, 实现生产指令的完成打下基础, 进而实现船舶 制造车间的精确化、柔性化生产，进而提高车间生 产效率。

\section{2 多工位协同系统模型研究}

多工位协同系统模型研究是开展多工位协同制 造技术系统的基础, 建模过程中需要考虑的关键技 术点是: (1) 在分析车间生产的众多关联因素的基础 上，挖掘影响多工位间协同作业的关键参量; (2) 基 于船舶车间的生产工艺及生产模式，明确协同目标 并分析各参量间的关联关系; (3) 分析各因素对协同 目标的影响程度，建立多工位协同的动态调整机制。 通过模型的建立，实现船舶制造车间各层次对任务、 资源、时间三要素的高效协作，保证车间的正常生 产。建模前首先分析船舶分段制造车间的具体组成, (图 1)。船舶分段制造车间由多个工位组成, 其中各 生产工位包含了多道子加工工序, 通过梳理车间结 构, 进而分析多工位协同的整体流程。

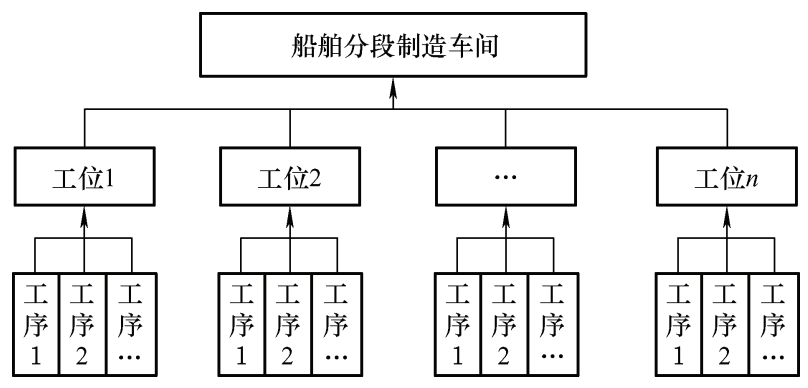

图 1 船舶分段车间组成

\section{1 多工位协同制造模型主要影响因素分析}

船舶分段制造生产过程中存在的不确定的因 素 ${ }^{[1-12]}$, 会导致实际生产过程与计划间存在较大差 异。以实际生产为例, 生产中会出现物料短缺、工 具缺位、人员变更等复杂、紧急情况, 导致执行进 度在工位、工序间出现偏差, 影响生产效率。如中 
小组立车间工位, 在实际加工过程中需要上道工位/ 工序提供加工物料, 一旦出现物料供应不足, 则导 致该工位出现临时停工现象, 进而影响后道工位的 生产加工。生产过程中可能影响生产效率的相关因 素，如表 1 所示。

结合生产制造过程影响因素分析, 采用自动协 同和手动协同机制开展多工位协同建模研究。以船舶 制造工艺数据模型和多工序间的数据驱动模型为基 础, 结合船舶建造车间的钢板切割、板材理料、坡口 加工、材料配送以及中小组立等车间工位的加工特 点, 研究现场多工位多工序的计划完工日期、目标工 时、计划分配等之间的协同作业流程和交互关系, 建 立多工位多工序间协同模型, 为集中管控的精准化管 理提供支撑。协同动态调整流程如图 2 所示。
表 1 因素分析

\begin{tabular}{cl}
\hline \multicolumn{1}{c}{ 种类 } & \multicolumn{1}{c}{ 因素分析 } \\
\hline & 生产资源短缺、延迟 \\
& 紧急插单 \\
& 预计交货期的改变/订单取消 \\
& 任务计划顺影响调整 \\
\hline & 临时工艺 \\
& 工艺/物量更改 \\
& 工件质量问题返工 \\
\hline & 设备维修 \\
& 设备故障 \\
& 作业人数变化/生产人员作业能力 \\
& 作业时间变化 \\
\hline 生产能力影响 & 计划与实际生产时间的差距 \\
\hline
\end{tabular}

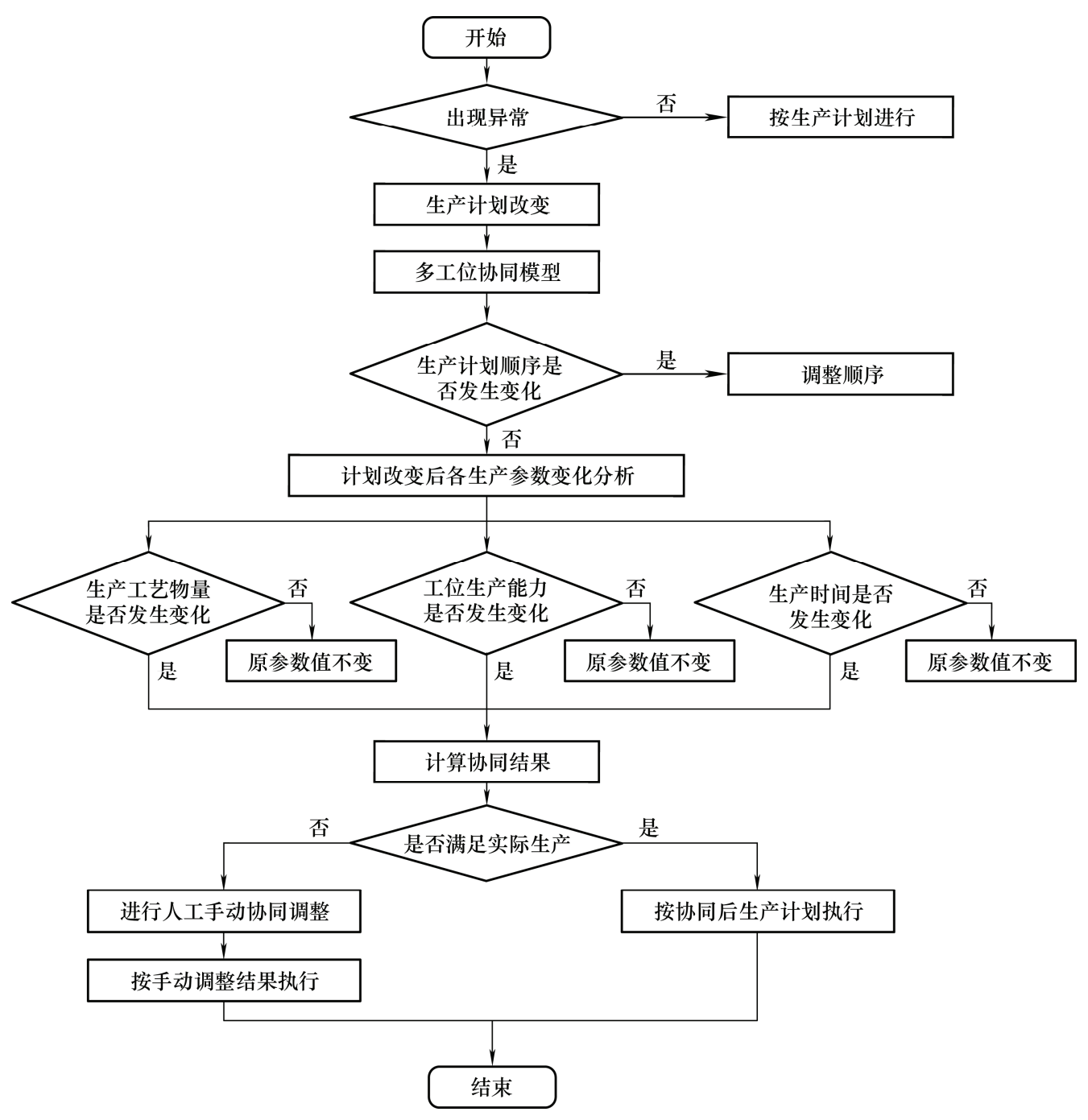

图 2 多工位协同动态调整流程图

\section{2 多工位协同数学模型建立}

以船厂某日常月的分段作业计划为输入, 以最 短生产时间为约束, 分析各工位效率, 提出最佳协 同作业方法, 解决目前生产中不同工位间存在的执
行偏差问题, 以达到生产过程中资源与设备分配趋 于均衡, 实现生产计划高效执行, 提高生产效率。 船舶制造多工位协同目的是实现分段制造过程 多工位设备高效工作, 特别是在计划调整, 插单、 
设备故障时, 对生产任务加工顺序, 加工时间等动 态调整及监控。

基于船舶分段制造过程中主要影响因素, 建立 多工位协同数学模型

$$
\begin{gathered}
\min T=F(J, P, C, S T, E T, \Theta) \\
\text { s.t. } S T_{i j}-E T_{i(j-1)} \geqslant 0 \\
S T_{i j}-E T_{(i-1) j} \geqslant 0 \\
C \geqslant 0
\end{gathered}
$$

式中 $T$ 一工位级生产任务总工期;

$J$ 一车间工位集;

$P$ 一月度生产任务集;

$C$ 一各工位作业能力;

$S T$ 一作业计划开始时间;

$E T$ 一作业计划完工时间;

$\Theta$ 一实际作业过程中出现的其他异常情况 参数。如生产工艺/工序、作业人数、 业务水平、作业时间、设备状态等各

\subsection{1 车间工位集 $J$} 类参数变化对其结果均可产生影响。

生产制造过程最小设备工作单元是工位。多工 位协同的基础单元也设定为工位。实际制造过程中, 存在一个工艺一个工位, 一个工艺多个工位、多工 艺多工位的情况, 工位集是按照实际生产过程、生 产工艺等划分工位的集合，可表示为

$$
J=\{j \in J \mid j=1,2, \cdots, n\}
$$

结合不确定情况分析, 如果出现增加工位或撤 销工位等情况时, 可根据实际情况对工位集合进行 修改、维护，提高工位集的柔性管理。

\subsection{2 月度生产任务集 $P$}

船舶分段制造过程生产计划逐级包括年度生产 计划、季度生产计划和月度生产计划。现有车间的 生产计划仅下达到月度计划, 计划颗粒度大, 考虑 到计划与实际设备执行存在的差异, 包括由各种原 因造成的生产计划完成误差(如紧急插单、资源不足 等)、实际生产变化引起的预测误差等。因此, 需要 将月度生产计划按生产任务进一步分配到车间工位 级生产任务, 同时确定各工位生产任务的加工物量。 结合实际生产流程, 从生产任务和生产物量两个维 度进行月度生产任务拆解, 如图 3 所示。

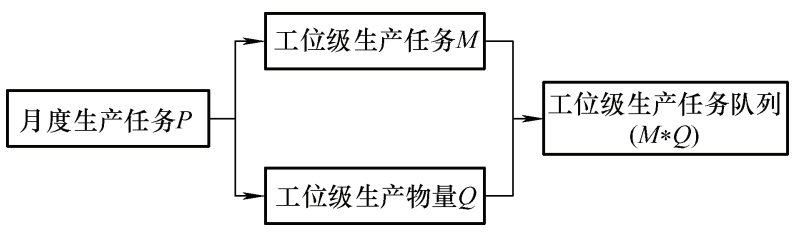

图 3 生产任务拆解流程
(1) 工位级生产任务。

假设, 月度生产计划中的总生产任务为 $P=$ $\left\{P_{1}, P_{2}, P_{3}, \cdots, P_{i}\right\}$, 其中 $i$ 为正整数, 代表生产任务数量。

$M_{i}=\left\{M_{i 1}, M_{i 2}, M_{i 3}, \cdots, M_{i j}\right\}$ 表示月度生产任务 $P_{i}$ 中对应的各工位生产任务, 其中, $j \in J$ 代表月度 生产任务 $P_{i}$ 中所需的生产工位数量。

因此, 工位级生产任务 $M$ 表示为

$$
\begin{array}{r}
\boldsymbol{M}=\left[\begin{array}{ccccc}
M_{1} & M_{2} & M_{3} & \cdots & M_{i}
\end{array}\right]^{\mathrm{T}}= \\
\\
{\left[\begin{array}{ccccc}
M_{11} & M_{12} & M_{13} & \cdots & M_{1 j} \\
M_{21} & M_{22} & M_{23} & \cdots & M_{2 j} \\
M_{31} & M_{32} & M_{33} & \cdots & M_{3 j} \\
\vdots & \vdots & \vdots & & \vdots \\
M_{i 1} & M_{i 2} & M_{i 3} & \cdots & M_{i j}
\end{array}\right]}
\end{array}
$$

(2) 工位级生产物量。

根据图 1 可知，船舶分段制造过程中，每个工 位生产任务包含多道子加工工序, 各工位生产任务 对应子加工工序的加工物量来源于生产任务物料清 单，分析月度生产计划和物料清单可以得到各生产 任务所在工位的加工物量 $Q$ 。

假设， $Q_{i}=\left\{Q_{i 1}, Q_{i 2}, Q_{i 3}, \cdots, Q_{i j}\right\}$ ，表示月度生产 任务 $P_{i}$ 中对应的 $j$ 个工位的生产任务的加工物量。

因此, 工位级生产物量 $\boldsymbol{Q}$ 表示为

$$
\begin{array}{r}
\boldsymbol{Q}=\left[\begin{array}{lllll}
Q_{1} & Q_{2} & Q_{3} & \cdots & Q_{i}
\end{array}\right]^{\mathrm{T}}= \\
{\left[\begin{array}{ccccc}
Q_{11} & Q_{12} & Q_{13} & \cdots & Q_{1 j} \\
Q_{21} & Q_{22} & Q_{23} & \cdots & Q_{2 j} \\
Q_{31} & Q_{32} & Q_{33} & \cdots & Q_{3 j} \\
\vdots & \vdots & \vdots & & \vdots \\
Q_{i 1} & Q_{i 2} & Q_{i 3} & \cdots & Q_{i j}
\end{array}\right]}
\end{array}
$$

式中, 生产任务 $P_{i}$ 在第 $j$ 个工位的生产物量 $Q_{i j}$ 是由 该工位的 $k$ 道子加工工序的物量组成, 各子工序物 量可表示为 $Q_{i j}=\left\{q_{i j 1}, q_{i j 2}, q_{i j 3}, \cdots, q_{i j k}\right\} ， k$ 取值 $1 \leqslant k \leqslant z$, 则各任务在各工位的加工物量为

$$
Q_{i j}=\sum_{k=1}^{z} \delta_{k} q_{i j k}
$$

式中, $\delta_{k}$ 表示子工序加工物量变化系数, 可结合船 企实际作业中各子工序物量变化系数按工序难易程 度及生产经验取值。当出现临时工艺或工艺更改等 异常情况时, 通过更新 $\delta_{k}$ 系数实现物量参数的修 改, 进而改变 $Q_{i j}$ 的值。

根据得到的工位级生产任务 $J$ 以及各任务对应 工位的生产物量 $Q$, 进一步将各工位的生产任务和 所对应生产物量进行组合，最终得到月度生产计划 中的总生产任务 $P$ 拆解到工位级的详细生产任务, 其拆解结果采用哈达马积进行表示 


$$
\begin{gathered}
{\left[\begin{array}{ccccc}
M_{11} & M_{12} & M_{13} & \cdots & M_{1 j} \\
M_{21} & M_{22} & M_{23} & \cdots & M_{2 j} \\
M_{31} & M_{32} & M_{33} & \cdots & M_{3 j} \\
\vdots & \vdots & \vdots & \ddots & \vdots \\
M_{i 1} & M_{i 2} & M_{i 3} & \cdots & M_{i j}
\end{array}\right] *\left[\begin{array}{ccccc}
Q_{11} & Q_{12} & Q_{13} & \cdots & Q_{1 j} \\
Q_{21} & Q_{22} & Q_{23} & \cdots & Q_{2 j} \\
Q_{31} & Q_{32} & Q_{33} & \cdots & Q_{3 j} \\
\vdots & \vdots & \vdots & \ddots & \vdots \\
Q_{i 1} & Q_{i 2} & Q_{i 3} & \cdots & Q_{i j}
\end{array}\right]} \\
{\left[\begin{array}{ccccc}
M_{11} \cdot Q_{11} & M_{12} \cdot Q_{12} & M_{13} \cdot Q_{13} & \cdots & M_{1 j} \cdot Q_{1 j} \\
M_{21} \cdot Q_{21} & M_{22} \cdot Q_{22} & M_{23} \cdot Q_{23} & \cdots & M_{2 j} \cdot Q_{2 j} \\
M_{31} \cdot Q_{31} & M_{32} \cdot Q_{32} & M_{33} \cdot Q_{33} & \cdots & M_{3 j} \cdot Q_{3 j} \\
\vdots & \vdots & \vdots & \vdots & \vdots \\
M_{i 1} \cdot Q_{i 1} & M_{i 2} \cdot Q_{i 2} & M_{i 3} \cdot Q_{i 3} & \cdots & M_{i j} \cdot Q_{i j}
\end{array}\right]}
\end{gathered}
$$

当出现紧急插单、计划变更等生产扰动时, 只 需将相应的批次任务插入到月度生产任务 $P$ 中, 进 行模型重新计算。

例如, 插单任务为 $P_{x}$, 此时只需修改月度生产任 务集 $P$, 实现紧急插单, 如 $P=\left\{P_{1}, P_{2}, P_{3}, \cdots, P_{x}, \cdots, P_{i}\right\}$, 同时进行工位级生产任务和生产物量拆解得到最终 工位级生产任务队列。

\subsection{3 各工位作业能力集}

船舶分段制造过程涉及的工位可分为两种, 包 括手动工位(班组作业)和自动工位(设备作业)。作业 能力是指各工位在单位时间内可以加工的物量多 少, 以纵骨焊接工位为例, 该工位的作业能力约为 $45 \mathrm{~m} / \mathrm{h}$ 。

假设, 各工位作业能力集 $C$ 表示为

$$
C=\left\{C_{1}, C_{2}, \cdots, C_{j}\right\}, j \in J
$$

式中, $C_{1}$ 表示手动工位作业能力; $C_{2}$ 表示自动工位 作业能力; 则各工位对应的作业能力表示为

$$
\begin{gathered}
C_{j}=\lambda_{1} \delta_{1} c_{1}+\lambda_{2} \delta_{2} c_{2} \\
\lambda_{1}, \lambda_{2}=\left\{\begin{array}{l}
0 \\
1
\end{array}\right.
\end{gathered}
$$

式中, $\lambda_{1}=1$ 表示为手动工位; $\lambda_{2}=1$ 表示为自动工 位; $\delta_{1}$ 表示手动工位作业能力系数; $\delta_{2}$ 表示自动工 位作业能力系数。

工位作业能力属于不确定参数, 与作业人员的 数量 $n$ 、业务水平 $v$ 、作业时间 $t$ 、设备状态 $s$ 等均 有关系, 当其中一个变量发生变化, 对应的作业能 力系数随之改变, 表示为

$$
\delta_{1}, \delta_{2}=f(n, v, t, s)
$$

自动工位作业能力可根据设备加工能力进行估 算, 手动工位作业能力可根据现场工作人员的经验 给出相关参数。

当出现人员变更、设备故障等生产扰动时, 将 更新后的相关变量参数带入模型重新计算。

\subsection{4 作业计划开始时间和作业计划完工时间}

各工位的作业开始时间、完工时间的计算，主 要结合任务生产物量、工位作业能力以及生产任务 序列进行计算。其中, $S T_{i j}$ 表示第 $i$ 个生产任务在工 位 $j$ 的计划开始时间; $E T_{i j}$ 表示第 $i$ 个生产任务在工 位 $j$ 的计划完工时间; $\widetilde{S T}_{i j}$ 表示第 $i$ 个生产任务在工 位 $j$ 的实际开始时间, 由监控系统进行反馈; $\widetilde{E T}_{i j}$ 表 示第 $i$ 个生产任务在工位 $j$ 的实际完工时间, 由监控 系统进行反馈; $T_{i j}$ 表示第 $i$ 个生产任务在工位 $j$ 的 计划工时。

则各工位生产任务的计划工时可表示为

$$
T_{i j}=\frac{Q_{i j}}{C_{j}}=\frac{\sum_{k=1}^{z} \delta_{k} q_{i j k}}{\lambda_{1} \delta_{1} C_{1}+\lambda_{2} \delta_{2} C_{2}}
$$

通过分析可知, 在同一工位, 下道任务必须在 上道任务完工之后才能开始，同时，下道工位的任 务加工必须在上道工位任务完工后才能开始, 因此, 任务加工顺序必须满足以下条件

$$
\left\{\begin{array}{l}
S T_{i j} \geqslant E T_{i(j-1)} \\
S T_{i j} \geqslant E T_{(i-1) j}
\end{array}\right.
$$

任务的计划总工期则表示为

$$
T=\sum_{i=1}^{m} \sum_{j=1}^{n}\left\{\left(1+\delta_{1}\right) E T_{i j}-\left(1+\delta_{2}\right) S T_{i j}\right\}
$$

式中, $\delta_{1}$ 为任务的实际开始时间与计划开始时间的 差异系数; $\delta_{2}$ 为任务的实际完工时间与计划完工时 间的差异系数。系数计算方法如下

$$
\left\{\begin{array}{l}
\delta_{1}=\frac{\widetilde{E T}_{i j}-S T_{i j}}{S T_{i j}} \\
\delta_{2}=\frac{\widetilde{E T}_{i j}-E T_{i j}}{E T_{i j}}
\end{array}\right.
$$

当实际生产过程中出现实际作业时间与计划时 间的偏差时, 通过监控系统采集的实际数据进行差 异系数 $\delta_{1} 、 \delta_{2}$ 的计算, 将参数引入模型重新计算, 实现生产计划的动态协同调整。

\section{3 典型应用验证分析}

在船舶制造实际作业过程中, 典型需要开展协 同作业就是任务临时变化, 本文以实际生产过程中 出现紧急插单情况作为典型异常情况进行分析讨 论。针对出现的紧急插单这类生产扰动, 结合图 1 进行分析, 紧急插单对生产计划、生产工艺物量、 生产时间均会产生影响, 进一步结合具体的数学模 
型对各参数进行计算, 完成紧急插单异常下的多工 位协同过程。

以某船厂分段加工车间为对象进行验证分析, 制造车间共涉及预拼板, 拼板, FCB 焊接修补, 纵 骨装配 5 个工艺, 6 个工位, 主要为: 预拼工位 J1、 拼板工位 J2、FCB 焊接工位 J3、修补工位 J4、纵骨 装配工位 J5 及纵骨装配工位 J6, 则拆解得到车间工 位集 $\mathrm{J}=\{\mathrm{J} 1, \mathrm{~J} 2, \mathrm{~J} 3, \mathrm{~J} 4, \mathrm{~J} 5, \mathrm{~J} 6\}$ 。

假设车间按照正常生产计划, 现有 7 个待加工 的分段为: S1、S2、S3、S4、S5、S6、S7, 则月度 生产任务集 $P=\{\mathrm{S} 1, \mathrm{~S} 2, \mathrm{~S} 3, \mathrm{~S} 4, \mathrm{~S} 5, \mathrm{~S} 6, \mathrm{~S} 7\}$ 。

根据某月度生产计划可知各分段的生产过程均 涉及 6 个工位, 因此, 将某月度生产任务带入文中 模型, 结合式(3) (6)进行工位级生产任务拆解, 确 定各分段任务所在工位对应的加工物量

$$
\boldsymbol{Q}=\left[\begin{array}{cccccc}
0 & 30.4 & 26.6 & 73.15 & 96.5 & 100 \\
0 & 30.4 & 26.6 & 73.15 & 96.5 & 100 \\
418.8 & 78.8 & 53.2 & 194.3 & 233.6 & 273 \\
462.85 & 96 & 108 & 285 & 227.3 & 440 \\
462.85 & 96 & 108 & 285 & 227.3 & 440 \\
0 & 138.8 & 95.2 & 341.8 & 172.8 & 291 \\
686 & 30.4 & 26.6 & 73.15 & 233.6 & 223
\end{array}\right]
$$

形成工位级生产任务数据如表 2 所示。

表 2 插单前工位级生产任务数据

\begin{tabular}{ccccccc}
\hline \multirow{2}{*}{ 任务 } & \multicolumn{5}{c}{ 工位 } \\
\cline { 2 - 7 } & $\mathrm{J} 1$ & $\mathrm{~J} 2$ & $\mathrm{~J} 3$ & $\mathrm{~J} 4$ & $\mathrm{~J} 5$ & $\mathrm{~J} 6$ \\
\hline $\mathrm{S} 1$ & 0 & 30.4 & 26.6 & 73.15 & 96.5 & 100 \\
$\mathrm{~S} 2$ & 0 & 30.4 & 26.6 & 73.15 & 96.5 & 100 \\
S3 & 418.8 & 78.8 & 53.2 & 194.3 & 233.6 & 273 \\
S4 & 462.85 & 96 & 108 & 285 & 227.3 & 440 \\
S5 & 462.85 & 96 & 108 & 285 & 227.3 & 440 \\
S6 & 0 & 138.8 & 95.2 & 341.8 & 172.8 & 291 \\
S7 & 686 & 30.4 & 26.6 & 73.15 & 233.6 & 223 \\
\hline
\end{tabular}

当出现紧急插单异常情况, 假设插单任务为 $\mathrm{S} 8$, 修改月度生产任务集 $P=\{\mathrm{S} 1, \mathrm{~S} 2, \mathrm{~S} 8, \mathrm{~S} 3, \mathrm{~S} 4, \mathrm{~S} 5, \mathrm{~S} 6, \mathrm{~S} 7\}$ 。 分段任务 S8 的生产过程均涉及 6 个工位, 同理, 结 合式(3) (6)进行工位级生产任务拆解, 确定各分段 任务所在工位对应的加工物量

$$
\boldsymbol{Q}=\left[\begin{array}{cccccc}
0 & 30.4 & 26.6 & 73.15 & 96.5 & 100 \\
0 & 30.4 & 26.6 & 73.15 & 96.5 & 100 \\
410.45 & 78.8 & 53.2 & 194.3 & 233.6 & 273 \\
418.8 & 78.8 & 53.2 & 194.3 & 233.6 & 273 \\
462.85 & 96 & 108 & 285 & 227.3 & 440 \\
462.85 & 96 & 108 & 285 & 227.3 & 440 \\
0 & 138.8 & 95.2 & 341.8 & 172.8 & 291 \\
686 & 30.4 & 26.6 & 73.15 & 233.6 & 223
\end{array}\right]
$$

形成工位级生产任务数据如表 3 所示。

表 3 插单后工位级生产任务数据

\begin{tabular}{ccccccc}
\hline \multirow{2}{*}{ 任务 } & \multicolumn{7}{c}{ 工位 } \\
\cline { 2 - 7 } & J1 & J2 & J3 & J4 & J5 & J6 \\
\hline S1 & 0 & 30.4 & 26.6 & 73.15 & 96.5 & 100 \\
S2 & 0 & 30.4 & 26.6 & 73.15 & 96.5 & 100 \\
S8 & $\mathbf{4 1 0 . 4 5}$ & $\mathbf{7 8 . 8}$ & $\mathbf{5 3 . 2}$ & $\mathbf{1 9 4 . 3}$ & $\mathbf{2 3 3 . 6}$ & $\mathbf{2 7 3}$ \\
S3 & 418.8 & 78.8 & 53.2 & 194.3 & 233.6 & 273 \\
S4 & 462.85 & 96 & 108 & 285 & 227.3 & 440 \\
S5 & 462.85 & 96 & 108 & 285 & 227.3 & 440 \\
S6 & 0 & 138.8 & 95.2 & 341.8 & 172.8 & 291 \\
S7 & 686 & 30.4 & 26.6 & 73.15 & 233.6 & 223 \\
\hline
\end{tabular}

进一步, 通过确定实际生产中各工位作业能力、 计算作业工时等, 结合式(7) (12)进行生产总工时 计算, 分析计算可得以下几点。

(1) 当不存在相关影响时, 采用船舶分段制造 多工位协同管控模型对车间的 7 个待加工生产任务 进行工位级计划拆解, 结合船厂分段加工车间各工 位的实际生产作业能力, 采用式(10)计算各工位生 产任务的计划工时, 得到各生产任务在工位级的生 产计划如图 4 所示, 生产总工时为 $110.95 \mathrm{~h}$ 。

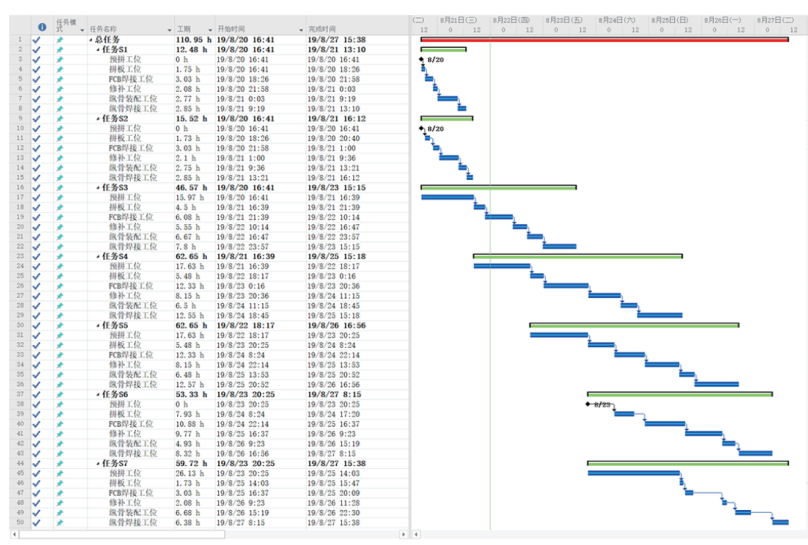

图 4 正常生产计划

(2) 当出现紧急任务 S8 时如上表 2 所示, 采用 普通的插单处理, 根据任务紧急程度以及实际生产 进度将任务插入计划列表, 假设 S8 的紧急程度较 大, 实际生产进行到 $\mathrm{S} 2$, 则插入任务后的分段加 工顺序为 $\mathrm{S} 1 \rightarrow \mathrm{S} 2 \rightarrow \mathrm{S} 8 \rightarrow \mathrm{S} 3 \rightarrow \mathrm{S} 4 \rightarrow \mathrm{S} 5 \rightarrow \mathrm{S} 6 \rightarrow \mathrm{S} 7$, 处 理后的生产计划更新如图 $5 \mathrm{a}$ 所示, 生产总工时为 $126.58 \mathrm{~h}$ 。

(3) 采用多工位协同控制系统进行插单处理, 协同处理后生产任务的分段加工顺序为 $\mathrm{S} 1 \rightarrow \mathrm{S} 2 \rightarrow$ $\mathrm{S} 8 \rightarrow \mathrm{S} 3 \rightarrow \mathrm{S} 5 \rightarrow \mathrm{S} 6 \rightarrow \mathrm{S} 4 \rightarrow \mathrm{S} 7$, 生产计划如图 $5 \mathrm{~b}$ 所示, 生产总工时为 $123.87 \mathrm{~h}$ 。

（4）对比普通插单处理和工位协同模型应用, 
计划总工时缩短 $2.71 \mathrm{~h}$, 提高了制造车间生产效率, 同时节约中间相关缓存量。

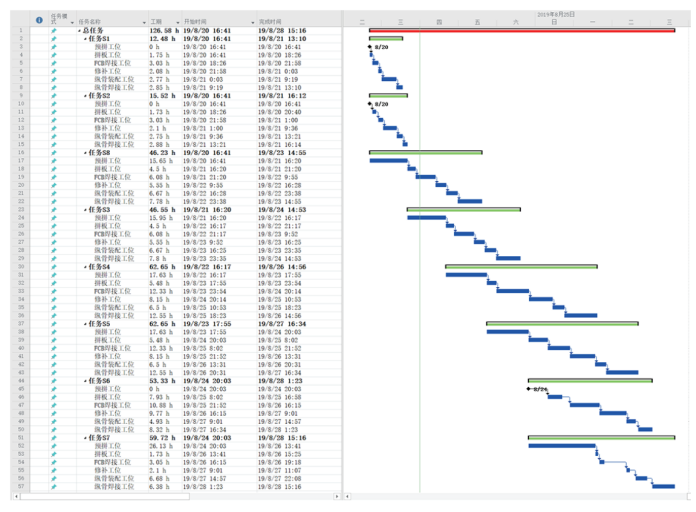

(a) 紧急插单生产计划

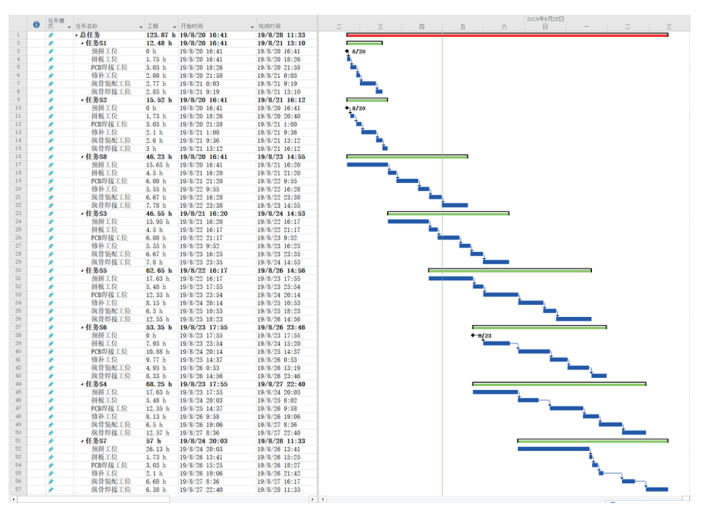

(b) 紧急插单后手动调整

图 5 紧急插单后手动调整的生产计划

\section{4 结论}

(1) 在对船舶制造行业的信息化分析的基础 上，提出船舶制造车间级的多工位协同制造系统的 概念。基于对传统车间生产模式和工位生产计划相 关因素分析, 研究了多工位协同动态调整机制, 建 立多工位协同系统数学模型。并以某生产分段紧急 插单情况为案例, 验证分析了多工位协同模型及动 态调整的有效性。

(2) 开展船舶多工位协同制造研究, 是构建互联 互通的船舶制造车间平台的重要环节之一, 有助于 推进船舶智能制造过程管控的技术提升, 促进船舶 数字化制造行业转型升级, 助力中国制造 2025 。

\section{参 考 文 献}

[1] 邸立强, 杨剑征, 赵川. 国外数字化造船技术发展趋势 研究[J]. 舰船科学技术, 2015, 37(7): 1-4.

DI Liqiang, YANG Jianzheng, ZHAO Chuan. Development of foreign digital shipbuilding technique[J]. Ship Science and Technology, 2015, 37(7): 1-4.
[2] 兰宏凯, 杨志, 柳存根, 等. 船舶平面分段流水线模糊 调度系统开发 $[\mathrm{J}]$. 船舶工程, 2018，40(11)：18-22.

LAN Hongkai, YANG Zhi, LIU Cungen, et al. Development of ship plane segmented line fuzzy scheduling system[J]. Ship Engineering, 2018，40(11): 18-22.

[3] HAMID S H , ZHANG Zhihai, OMID Z, et al. Trade-off between the costs and the fairness for a collaborative production planning problem in make-to-order manufacturing[J]. Computers \& Industrial Engineering, 2018, 126: $421-434$.

[4] 谢志强, 张晓欢, 高一龙, 等. 考虑串行工序紧密度的 择时综合调度算法[J]. 机械工程学报, 2018, 54(6): 191-202.

XIE Zhiqiang, ZHANG Xiaohuan, GAO Yilong, et al. Time-selective integrated scheduling algorithm considering the compactness of serial processes[J]. Journal of Mechanical Engineering, 2018， 54(6): 191-202.

[5] 苏翔, 孙红霞. 基于 MES 的造船企业车间作业调度系 统研究[J]. 造船技术, 2009(3): 23-27.

SU Xiang, SUN Hongxia. Research on job shop scheduling system of shipbuilding enterprise based on MES[J]. Marine Technology, 2009(3): 23-27.

[6] 张光发, 刘玉君, 纪卓尚. 船舶分段建造计划仿真与优 化[J]. 计算机集成制造系统, 2011，17(12): 2643-2651. ZHANG Guangfa, LIU Yujun, JI Zhuoshang. Simulation and optimization of ship block-building planning $[\mathrm{J}]$. Computer Integrated Manufacturing Systems, 2011, 17(12): 2643-2651.

[7] PAN Tianyi, ZHANG Zhihai, CAO Hui. Collaborative production planning with production time windows and order splitting in make-to-order manufacturing[J]. Computers \& Industrial Engineering, 2014, 67.

[8] KAMENG N, WANG Zhenbo, FABRICE T N, et al. A combination of flow shop scheduling and the shortest path problem[J]. Journal of Combinatorial Optimization, 2015, 29(1): 36-52.

[9] YU Yihao, WANG Yanhong. Design and implementation of a real-time Jobshop scheduling system[C]//2012 IEEE 19th International Conference on Industrial Engineering and Engineering Management(IE\&EM 2012).

[10] CAPUTO G. Optimization of production plan through simulation techniques[J]. WSEAS Transactions on Information Science and Application, 2009(6): 352-362.

[11] 王超, 刘阶萍, 常伟涛, 等. 不确定条件下的作业车间 生产调度综述[J]. 装备制造技术, 2011(4): 144-148, 154. WANG Chao, LIU Jieping, CHANG Weitao, et al. 
Summary of production scheduling of the work workshop under uncertain conditions $[\mathrm{J}]$. Equipment Manufacturing Technology, 2011(4): 144-148, 154.

[12] 张蕾. 复杂作业模式下车间动态调度问题的研究 [J]. 机械设计与制造工程，2013，42(3): 41-44.

ZHANG Lei. Study on the dynamic scheduling of the workshop in the complex working mode[J]. Mechanical
Design and Manufacturing Engineering, 2013, 42(3): 41-44.

作者简介: 张倩, 女, 1981 年出生, 硕士, 高级工程师。主要研究方向 为装备自动化控制技术及自动化成组成线技术。

E-mail: zhangqian82618@163.com

单忠德(通信作者), 男, 1970 年出生, 博士, 研究员, 博士研究生导师。主 要研究方向为数字化机械装备与先进成形制造技术、智能制造技术与装备。

E-mail: shanzd@cam.com.cn

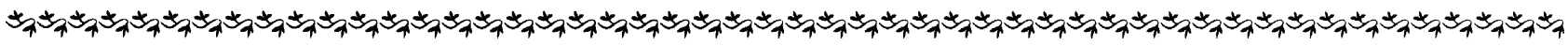

第 9 届上银优秀机械博士论文奖——佳作奖

\section{铝合金型材分流挤压焊合行为与焊缝质量控制方法研究}

作 者: 喻俊荃

毕业学校: 山东大学

指导教师: 赵国群

随着我国高速列车的快速发展, 高速列车车体用高性能大规格复杂截面空心铝合金型材的需求与日俱增。高速列车的高 速性、轻量化、舒适性和安全性等特点对高速列车车体铝合金型材的微观结构和性能, 尤其是焊缝质量, 提出了苛刻要求。 铝合金型材分流挤压焊合行为、界面结构演变规律与焊缝质量控制方法是制造高速列车用高性能大规格复杂截面空心铝合金 型材函待解决的关键科学和技术问题之一，也是国际上型材挤压成形领域面临的研究难题。

本文针对上述亟待解决的关键问题, 开展的主要研究工作和取得的研究成果如下。

(1) 提出了热压缩焊合与常规热压缩对比研究的新思路, 研究了铝合金材料的热变形和固态焊合行为。提出了一种表征 界面结合率的方法, 并建立了界面结合率与变形温度和应变速率的定量关系。提出了能够反映焊合界面结合程度的界面性能 特征参数。

(2) 设计并制造了一种结构参数可灵活调控的组合式分流挤压模具, 研究了纵向焊缝、横向焊缝和缩尾缺陷的微观结构、 抗腐蚀性能及其在拉伸过程中的断裂行为, 发现了应变集中和两种断裂模式。揭示了缩尾缺陷和横向焊缝的形成过程及机理。

(3) 开展了不同挤压模具结构参数下的挤压实验, 揭示了分流桥形状和焊合室深度对横向焊缝演变过程和长度的影响规 律以及对纵向焊缝形成过程、微观结构及力学性能的影响规律，提出了 “有效焊合面” 的概念。

(4) 研究了挤压速度和温度对挤压型材纵向焊缝的晶粒结构、界面结构、时效析出相以及力学性能的影响规律, 发现了 界面微孔洞和晶粒跨越界面生长现象。

(5) 研究了分流挤压过程中焊合路径上铝合金材料的晶粒结构和微观织构，揭示了其对型材力学性能的影响规律。

(6) 研究了挤压模具型腔内存在气孔和不存在气孔时焊合界面的精细结构, 发现了界面非晶层和界面迁移现象, 从微纳 尺度揭示了分流挤压过程中存在气孔和不存在气孔时分别对应的固态焊合机理。

(7) 研究了挤压焊合过程中纵向焊缝及宏观焊接缺陷的形成过程。提出了考虑应力三轴度、等效应变速率、温度和接触 时间的固态焊合准则。

(8) 提出了一种普适性的纵向焊缝焊合质量定量评估方法, 实现了非完全对称空心型材焊缝质量的数字化评估。 